\title{
Study of Multi-Beam Accelerator Driven Thorium Reactor
}

\author{
H. Ludewig, A. Aronson
}

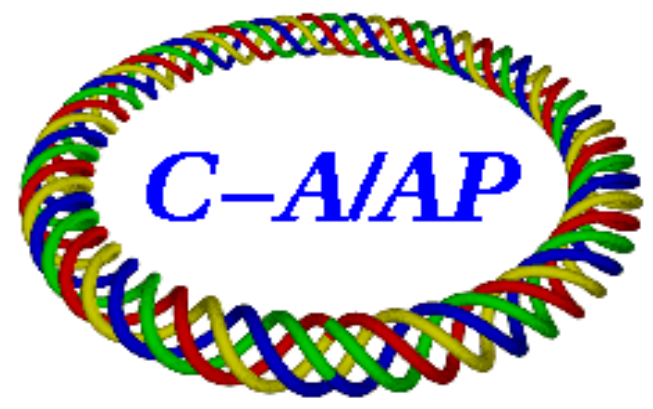

\section{Collider-Accelerator Department Brookhaven National Laboratory Upton, NY 11973}

Notice: This document has been authorized by employees of Brookhaven Science Associates, LLC under Contract No. DE-AC02-98CH10886 with the U.S. Department of Energy. The United States Government retains a nonexclusive, paid-up, irrevocable, world-wide license to publish or reproduce the published form of this document, or allow others to do so, for United States Government purposes. 


\title{
Study of Multi-Beam Accelerator Driven Thorium Reactor
}

\author{
H. Ludewig and A. Aronson
}

March 21, 2011

\section{A. Target/Blanket Assembly Description}

The primary advantages that accelerator driven systems have over critical reactors are:

1) Greater flexibility regarding the composition and placement of fissile, fertile, or fission product waste within the blanket surrounding the target, and

2) Potentially enhanced safety brought about by operating at a sufficiently low value of the multiplication factor to preclude reactivity induced events. The control of the power production can be achieved by vary the accelerator beam current. Furthermore, once the beam is shut off the system shuts down.

The primary difference between the operation of an accelerator driven system and a critical system is the issue of beam interruptions of the accelerator. These beam interruptions impose thermomechanical loads on the fuel and mechanical components not found in critical systems. Studies have been performed to estimate an acceptable number of trips, and the value is significantly less stringent than had been previously estimated. The number of acceptable beam interruptions is a function of the length of the interruption and the mission of the system. Thus, for demonstration type systems and interruption durations of $1 \mathrm{sec}<\mathrm{t}<5 \mathrm{mins}$, and $\mathrm{t}>5 \mathrm{mins} 2500 / \mathrm{yr}$ and $50 / \mathrm{yr}$ are deemed acceptable. However, for industrial scale power generation without energy storage type systems and interruption durations of $\mathrm{t}<1 \mathrm{sec}$., $1 \mathrm{sec}<\mathrm{t}<10$ secs., $10 \mathrm{secs}<\mathrm{t}<5 \mathrm{mins}$, and $\mathrm{t}>5 \mathrm{mins}$, the acceptable number of interruptions are 25000, 2500, 250, and 3 respectively. However, it has also been concluded that further development is required to reduce the number of trips. It is with this in mind that the following study was undertaken.

The primary focus of this study will be the merit of a multi-beam target system, which allows for multiple spallation sources within the target/blanket assembly. In this manner it is possible to ameliorate the effects of sudden accelerator beam interruption on the surrounding reactor, since the remaining beams will still be supplying source neutrons.

The proton beam will be assumed to have an energy of $1 \mathrm{GeV}$, and the target material will be natural lead, which will also be the coolant for the reactor assembly. Three proton beam arrangements will be considered, first a single beam (the traditional arrangement) with an entry at the assembly center, two more options will consist of three and six entry locations. The reactor fuel assembly parameters will be based on those of the S-PRISM fast reactor proposed by GE, and the fuel composition and type will be based on that proposed by Aker Solutions for use in their accelerator driven thorium reactor. The following table summarizes the parameters to be used in this study. The isotopic composition of the fertile material is $100 \%$ Th-232, and the plutonium isotopic distribution corresponds to that characteristic of the discharge from a typical LWR, following five years of decay. Thus, the isotopic distribution for the plutonium is; Pu-238 2.5\%, Pu-239 53.3\%, Pu-240 25.1\%, Pu-241 11.8\%, and Pu-242 $7.3 \%$. 
Table 1 - Parameters for the Accelerator Driven Thorium Reactor

\begin{tabular}{|c|c|}
\hline Parameter & Value \\
\hline Proton beam energy (GeV) & 1.0 \\
\hline Target Material/Coolant & $\mathrm{Pb}$ \\
\hline Fuel type and composition & $\mathrm{ThO}_{2} / \mathrm{PuO}_{2}-84.5 \% / 15.5 \%$ \\
\hline Plutonium isotopic vector & LWR discharge, following 5 year cool down \\
\hline Fuel smear density & $85 \%$ \\
\hline Fuel pellet radius $(\mathrm{cm})$ & 0.357 \\
\hline Clad inner radius $(\mathrm{cm})$ & 0.3875 \\
\hline Bond & $\mathrm{He}$ \\
\hline Clad outer radius $(\mathrm{cm})$ & 0.45 \\
\hline Clad material & HT9 \\
\hline Pin pitch/diameter, and arrangement & 1.2, hexagonal lattice \\
\hline Number of pins per assembly & 217 \\
\hline Active length $(\mathrm{cm})$ & 150.0 \\
\hline Fission gas plenum $(\mathrm{cm})$ & 170.0 \\
\hline Assembly duct inside flat-to-flat $(\mathrm{cm})$ & 15.9 \\
\hline Duct wall thickness $(\mathrm{cm})$ & 0.4 \\
\hline Duct gap $(\mathrm{cm})$ & 0.4 \\
\hline Assembly arrangement & Hexagonal lattice. \\
\hline
\end{tabular}

The number of hexagonal fuel assemblies in the reactor will vary, depending on the number and arrangement of the proton beam entry locations and size. For each arrangement, the number of assemblies will be varied until an initial value of the effective multiplication factor $\left(k_{e}\right)$ is approximately 0.95 .

The proton beam will be introduced into the target volume through a window that separates the vacuum of the drift tube from the flowing lead target. This window will need to withstand the material damage (displacements per atom (dpa)) due to the proton beam passing through it, and thus it is necessary to limit the proton current per unit area to practical limits. It is suggested that for this study the following assumptions be made in this regard.

1) In the case of a single entry location the middle seven assemblies be removed and used as the target. This would imply a circle with a radius of approximately $20 \mathrm{~cm}$ for the beam footprint on the window.

2) In the case of three entry locations a single fuel assembly can be removed, implying a window with a radius of approximately $8 \mathrm{~cm}$, and a third of the beam power.

3) Finally, in the case of six entry locations a single assembly will be removed, and the current density should be similar to that determined for the single entry location.

The suggested figures of merit to be used in this evaluation are:

1) The thermal stress induced in the ceramic fuel used in the thorium reactor following a sudden proton beam interruption from full power. In the case of a single beam the power interruption is 
more dramatic than in the cases with multiple entry locations. In the case with multiple entry locations, if one beam fails the remaining proton beams will still supply a source of spallation neutrons, and although the power shape will be skewed the power fluctuation will not be as dramatic. The implied thermal stress should not be as challenging for the fuel, and thus the figure of merit will be the value of the thermal stress and the expected fuel failure probability.

2) Variation of $\mathrm{k}_{\mathrm{e}}$ with time as the reactor operates and consumes the fissile material ( $\left.\mathrm{Pu}\right)$, and breeds in additional fissile material $\left(\mathrm{U}^{233}\right)$. If the value of $\mathrm{k}_{\mathrm{e}}$ becomes equal to a safe limit, external control will have to be imposed on the blanket. Alternatively a lower initial value of $k_{e}$ can be used. However, the latter strategy would imply a lower source multiplication, and thus a higher beam power for constant power output.

3) Variation of the required accelerator beam power to maintain a constant thermal power output from the reactor.

4) Window dpa, and gas production ( $\mathrm{H}$ and $\mathrm{He})$

In addition to the above figures of merit, two areas need to be addressed in a more complete study.

1) The above system is characterized by a fast neutron spectrum. Systems with both thermal and epi-thermal neutron spectra should also be investigated, particularly if $U^{233}$ is to be created from Th.

2) If a multi-beam arrangement is deemed necessary for reliable operation of the target/reactor, a complex mechanical arrangement is implied. It will thus be important to eventually address the overall target/reactor system reliability.

\section{B. Analysis method and modeling}

The analysis will be carried out primarily using the Monte Carlo code MCNPX. This code simulates the proton transport, spallation process, and subsequent determination of the reactor multiplication factor. The attached nuclear data library is used were applicable, and nuclear models internal to the code are used in the remaining energy ranges.

A model of the target and blanket was created to be consistent with the MCNPX input. Fig. 1 shows a section through a blanket assembly with 217 fuel rods. Fig. 2 shows a cross section through the target and blanket for the case using three proton beams. The fuel assemblies are arranged in a hexagonal lattice and the proton beams are introduced at three locations $120^{\circ}$ from each other. Fig. 3 shows a longitudinal section through the target. This section shows the proton beam drift tube, window, target, blanket, and surrounding reflector.

\section{Results of analysis}

A series of calculations was carried out with all the accelerator beams on and then repeated with one beam turned off. In this manner these calculations simulated the effect of a beam trip. In all cases the energy generated ( $\left.\mathrm{MeV} / \mathrm{gm}-\mathrm{p}^{+}\right)$in fuel assemblies located on a radius that passes through the tripped location was determined. Thus, the radial energy deposition before and after the beam trip can be determined, and therefore the fractional change in the various fuel assemblies can be computed. The fractional change around the tripped beam location will be of particular interest. In this analysis the assumption will be made that the thermo-mechanical load in the blanket zone is a function of the induced thermal stress. Furthermore, it will be assumed that: 
$\Delta \sigma$ (Change in thermal stress) $\alpha \Delta T$ (Change in temp.) $\alpha \Delta Q$ (Change in heat deposited)

Thus, to first order the change in thermal stress is directly proportional to the change in energy deposited in the fuel assembly, or any other component. The fractional change ((On-Off)/On) in energy deposited as a function of radius is given in the table below.

Table 2 - Fractional change in energy deposited as function of radius.

\begin{tabular}{|l|l|l|l|}
\hline Radial position $(\mathrm{cm})$ & \multicolumn{1}{|c|}{ One beam } & \multicolumn{1}{c|}{ Three beams } & \multicolumn{1}{c|}{ Six beams } \\
\hline 8.75 & & -0.00809 & -0.00096 \\
\hline 26.25 & & 0.123296 & 0.052654 \\
\hline 43.75 & 1.0 & 0.293227 & 0.139641 \\
\hline 61.25 & 1.0 & & \\
\hline 78.75 & 1.0 & 0.417357 & 0.216461 \\
\hline 96.25 & 1.0 & 0.388490 & 0.188398 \\
\hline 113.75 & 1.0 & 0.368829 & 0.163476 \\
\hline 131.25 & 1.0 & 0.339910 & 0.150387 \\
\hline
\end{tabular}

It is seen that with a single proton beam the maximum fractional change occurs, which is $100 \%$ at all locations. This induces the maximum stress possible in the various mechanical components including the fuel within the fuel rods. Introducing three beams, which implies three spallation neutron sources in the target/blanket assembly results in a maximum fractional change following one beam interruption is $41.7 \%$, and introducing six beams the maximum fractional change following a beam interruption is $21.6 \%$.

This reduction in the fractional change is essentially equal to the reduction in thermal stress experienced by the fuel and mechanical components of the target/blanket assembly.

In addition, the peak/average energy deposition is a measure of the overall power distribution within the blanket assembly. A lower value is desirable since the distribution of coolant flow is not as challenging, and the transmutation of fissile and fertile materials is spatially more uniform, thus involving more of the blanket. The peak/average for the single beam, three beam, and six beam configurations are $1.577,1.350$, and 1.358 respectively. A flatter distribution is obtained with a multibeam configuration.

\section{Summary}

The following summary and conclusions can be drawn from this study:

1) The fractional change in energy deposited in the fuel following beam trip is significantly reduced for the cases using multi-beam target arrangements. This implied reduction in thermal stress should lengthen the assembly mechanical life and reduce fatigue failures in general.

2) The production of spallation neutrons at multiple sites within the blanket reduces the peak/average energy deposition. This flatter distribution will ensure that the transmutation of the fissile and fertile materials takes place more uniformly, and that the coolant distribution among the assemblies does not change as much with time. 
Furthermore, it can be concluded that:

1) With multiple beam target arrangements the proton current impinging on the window is reduced relative to a single beam impinging on a single large window. It should thus be possible to design a window size that has a lower current density for multiple beam arrangements than for a single beam arrangement at constant total beam power. This should reduce the radiation damage in the window and thus increase window life.

2) With a flatter distribution implied for the multi-beam arrangements, more of the blanket is involved in the transmutation processes. This could affect the $k_{e} v s$. time characteristic of the target/blanket. If this characteristic is flatter then it would put a lower demand on the accelerator, since the required beam power variation with time will be lower.

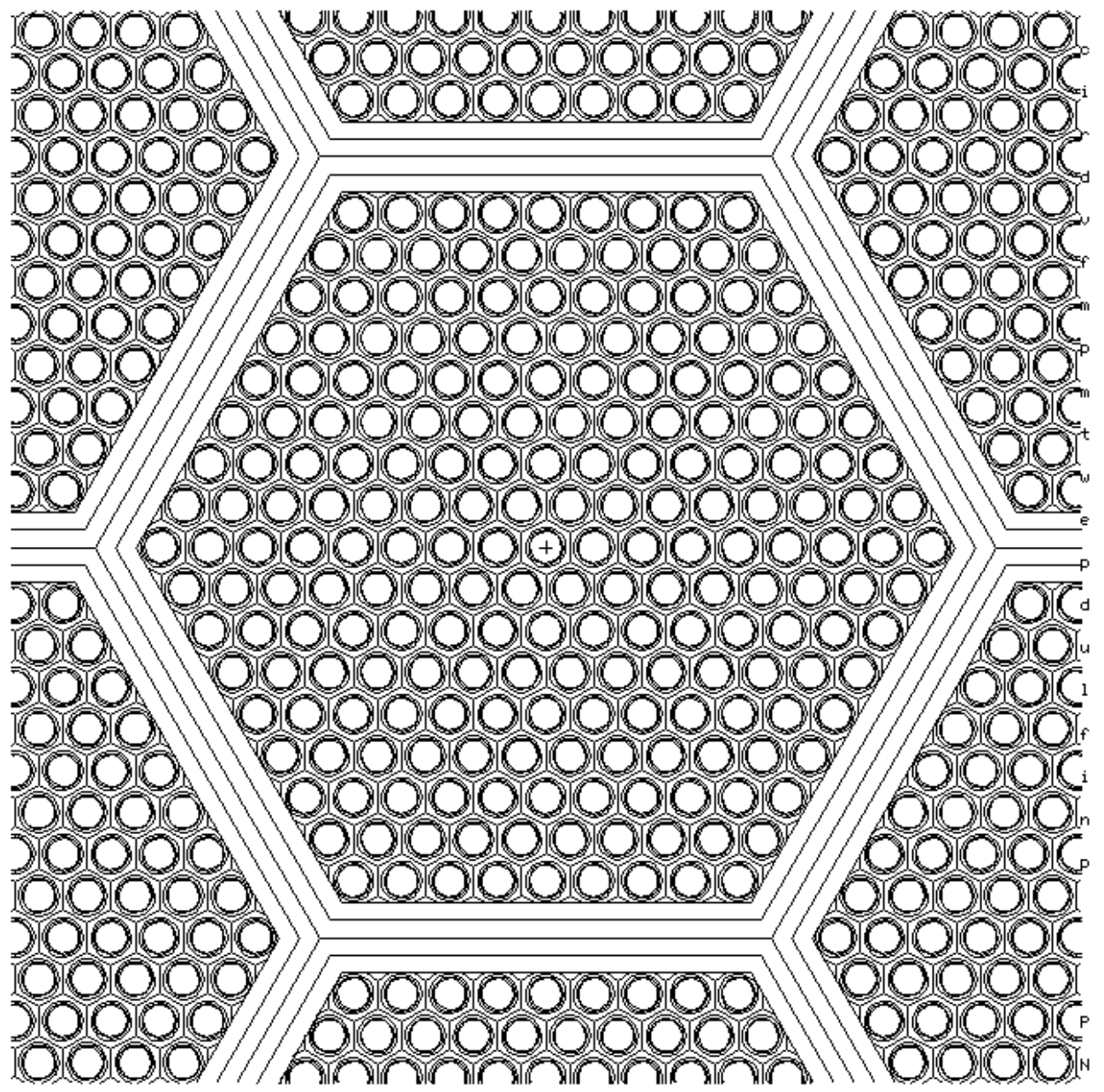

Fig. 1 - Detail of fuel assembly showing fuel rods, and hexagonal fuel assembly cans. 


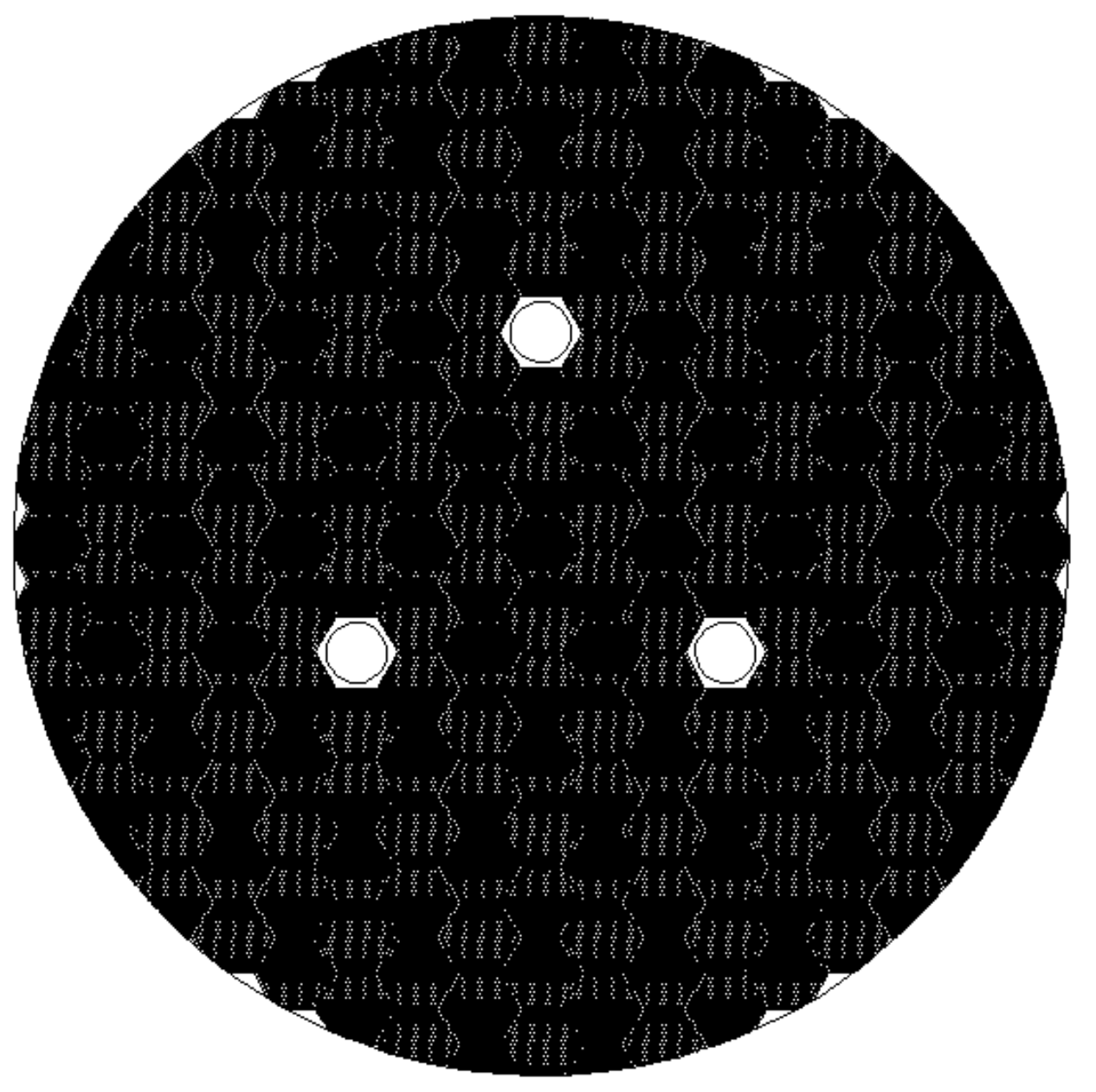

Fig. 2 - Section through target with three proton beams 


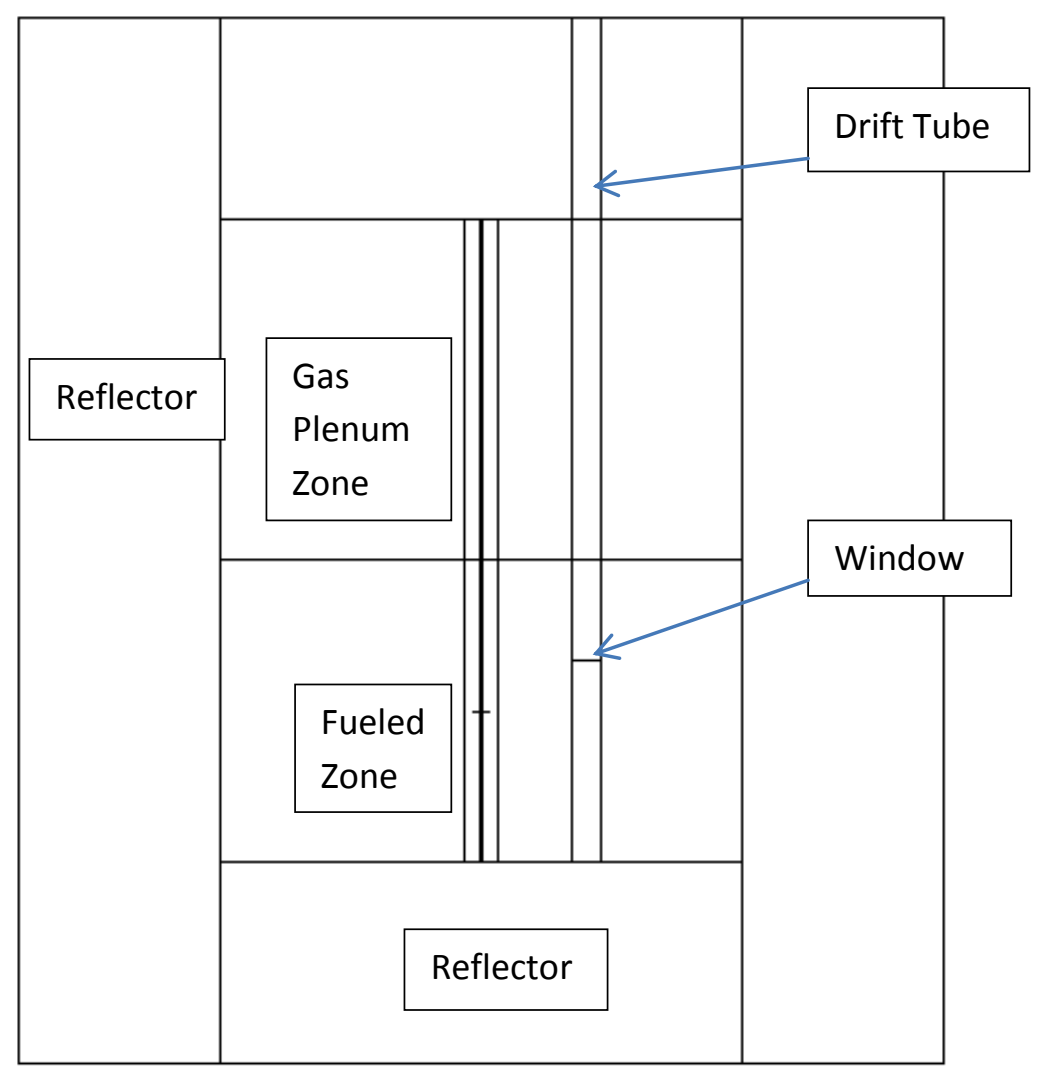

Fig. 3 - Longitudinal section through target showing one proton beam line and window. 